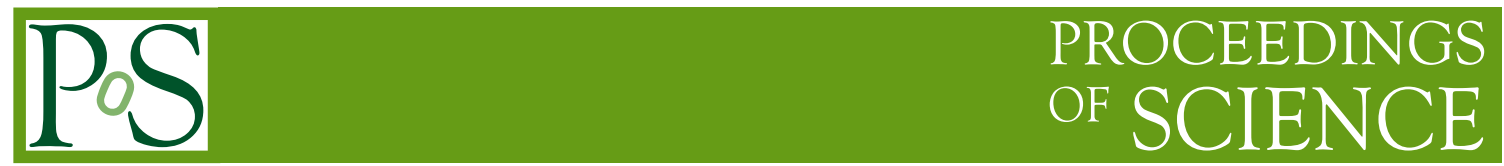

\title{
Beam Energy Dependence of Dielectron Production
}

\section{Xin Dong*}

Lawrence Berkeley National Laboratory

E-mail: XDongelbl.gov

I review recent experimental measurements of dielectron production in heavy ion collisions. Results reported by the RHIC experiments cover a wide range of collision energy from about top SPS energy up to the top RHIC energy. I will discuss very recent developments towards understanding the low mass enhancement difference between PHENIX and STAR. Finally I will argue the future prospectives of utilizing the RHIC beam energy scan to search for the onset of QGP thermal radiation.

8th International Workshop on Critical Point and Onset of Deconfinement,

March 11 to 15, 2013

Napa, California, USA

${ }^{*}$ Speaker. 


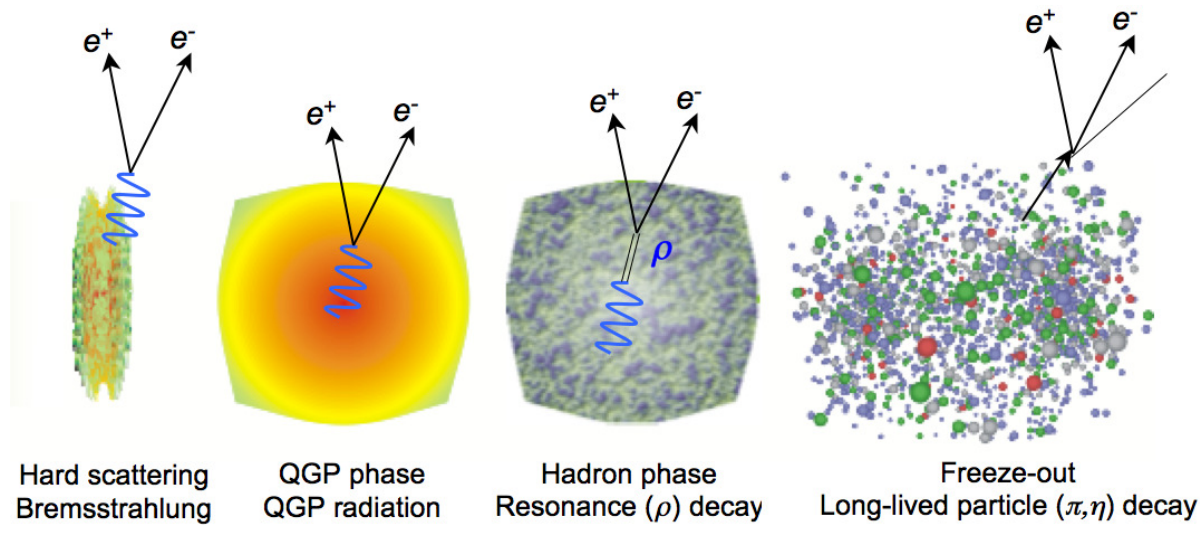

Figure 1: (Color online) Evolution of heavy ion collisions and dielectron sources from different stages.

\section{Introduction}

Dileptons, as they don't interact strongly, serve as a clean and penetrating probe to study the QCD medium created in heavy ion collisions. With the additional dimension of invariant mass, they offer an opportunity to investigate various stages of the medium. Figure 1 shows a schematic view of heavy ion collisions and the dielectron pair sources at different stages. Dielectrons produced in the partonic and hadronic medium are of particular interest for the study of medium properties as they are expected to be sensitive to the medium chiral symmetry and deconfinement $[1,2,3,4]$.

Dilepton measurements from the SPS data suggest the dileptons at low mass (mass below $\phi$ ) are dominated by the broadened $\rho$-meson interacting in the hadronic medium [5]. In this proceedings, I will review the recent experimental measurements at RHIC energies covering $\sqrt{s_{N N}}$ from 19.6 to $200 \mathrm{GeV}$.

\section{Dielectron Production at Top RHIC Energy}

Both PHENIX and STAR experiments at RHIC are equipped with excellent subsystems to carry out the dielectron measurements from $p+p$ to heavy ion collisions. Measurements from $p+p$ collisions from both demonstrated that the observed the dielectron yields can be described well by the hadronic cocktail $[6,7]$.

Figure 2 shows the dielectron invariant mass spectra in $\mathrm{Au}+\mathrm{Au}$ minimum bias collisions at $\sqrt{s_{\mathrm{NN}}}=200 \mathrm{GeV}$ measured in the PHENIX detector acceptance (left) [6] and the STAR detector acceptance (right) [8], respectively. Data points are compared to cocktail simulations and ratios of the data to cocktails are shown in the bottom panels. In the low mass region, both experiments observed an enhancement with respect to the cocktail and a vacuum $\rho$ spectral function cannot explain the excess. However, there appears to be a difference in the observed enhancement factor between two experiments. The enhancement factors with respect to the cocktail in the mass region of $0.15-0.75 \mathrm{GeV} / c^{2}$ in minimum bias collisions are $4.7 \pm 0.4 \pm 1.5$ from PHENIX and $1.53 \pm 0.07 \pm 0.41$ from STAR, respectively.

Figure 3 shows the low mass dielectron spectra measured by PHENIX and STAR compared to model calculations of the in-medium vector meson and QGP contributions from the same many- 

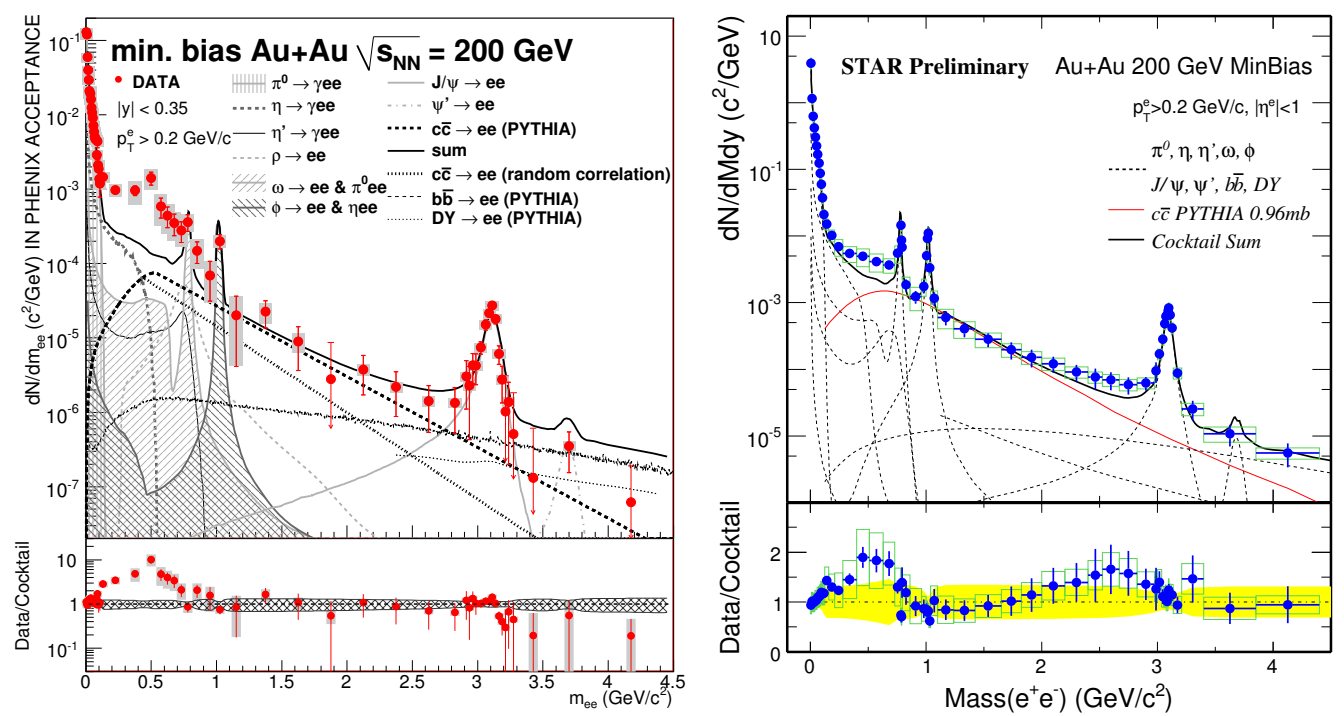

Figure 2: (Color online) Dielectron invariant mass distributions in $\mathrm{Au}+\mathrm{Au}$ minimum bias collisions at $\sqrt{s_{\mathrm{NN}}}=200 \mathrm{GeV}$ measured in the PHENIX detector acceptance (left) and the STAR detector acceptance (right), respectively. Data points are compared to cocktail simulations shown as different curves in the upper panels while the ratios of data to cocktail are shown in the bottom panels.

body effective theory in different detector acceptance cuts. In this model, the low mass excess is mainly attributed to the broadened $\rho$ spectral function due to the interactions in the hadronic medium [9]. Calculations from the same model with the similar hadronic medium have successfully explained the dilepton data measured at the SPS energies. The figure shows this model can reasonably reproduce the excess seen in the STAR data while under-predicts the data from PHENIX. Several other models show also they can explain the enhancement with the in-medium broadened $\rho$ contribution observed in the SPS and STAR data, but not the PHENIX data $[10,11,12]$.

\section{Experimental Efforts towards Understanding the Difference between PHENIX and STAR}

The difference in the low mass region in the observed dielectron data between PHENIX and STAR may be attributed to the different detector acceptances. It is expected that particle production at RHIC is roughly boost-invariant across $|y|<1$. Reducing the rapidity cut to fully reproduce the PHENIX acceptance with the STAR data is not likely to be significantly different while it will reduce the current STAR statistics by nearly a factor of 8 . Therefore, STAR tried to filter their data with the PHENIX azimuthal acceptance to understand this effect, and the resulting dielectron invariant mass spectrum is shown in Figure 4 for $\mathrm{Au}+\mathrm{Au} 200 \mathrm{GeV}$ minimum bias collisions. The result shows the enhancement factor w.r.t. the hadronic cocktail in the low mass region is still about a factor of 2, comparable with the STAR result without the azimuthal angle filtering. The difference in the low mass region between the PHENIX and the STAR results is unlikely due to the acceptance difference.

As both experiments calculated the enhancement factor with respect to each cocktail simulation baseline, it is natural to confirm that cocktail simulation of the two experiments are consistent. 

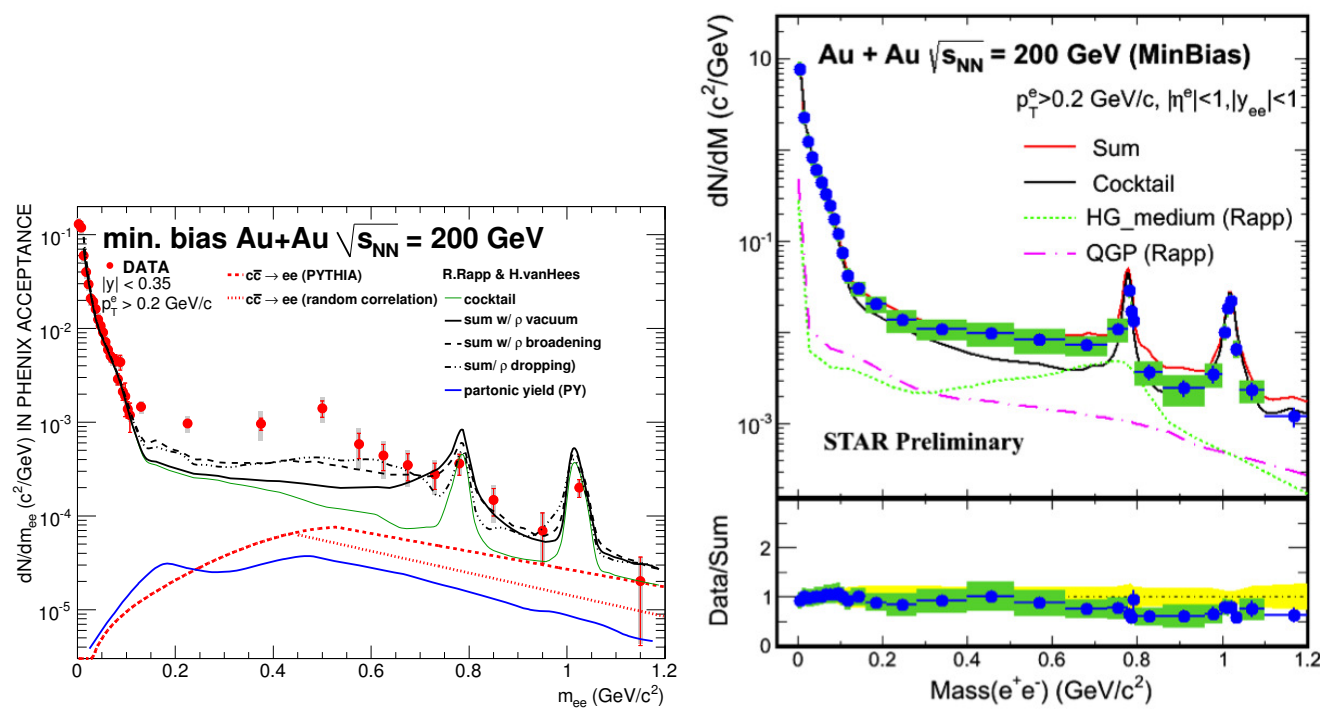

Figure 3: (Color online) Dielectron invariant mass spectra in the low mass region measured by PHENIX (left) and STAR (right). The spectra are compared to theoretical calculations of the in-medium vector meson and QGP contributions from the same many-body effective model with different detector acceptance cuts.

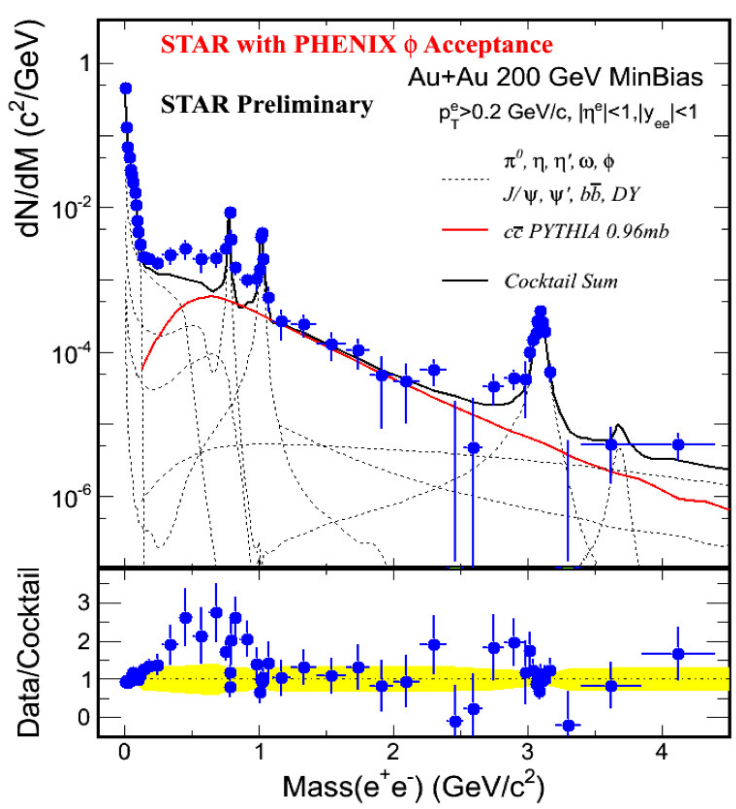

Figure 4: (Color online) Dielectron invariant mass spectra using the STAR data filtered with the PHENIX azimuthal acceptance. 


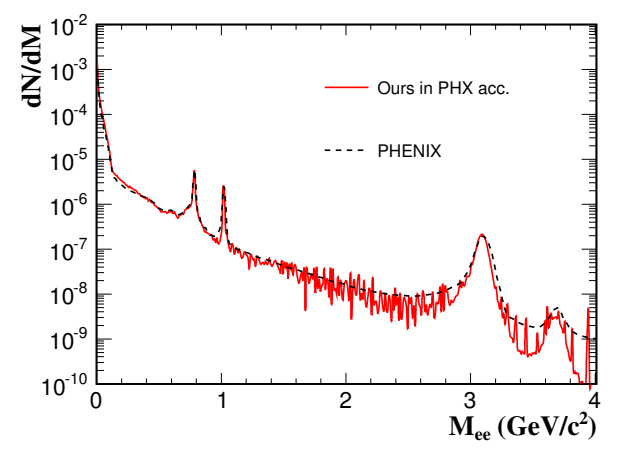

Figure 5: (Color online) Comparison of the total cocktail sum within the PHENIX azimuthal angle acceptance calculated by STAR (red solid) and PHENIX (black dashed) for $p+p 200 \mathrm{GeV}$ collisions

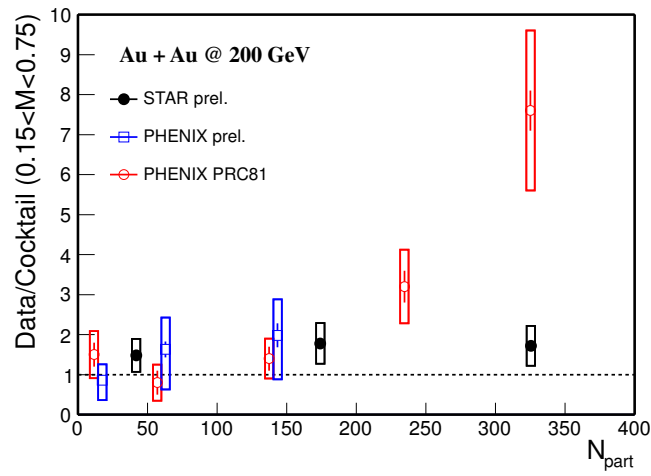

Figure 6: (Color online) Centrality dependence of the dielectron enhancement factor w.r.t. the cocktail in the mass region of $0.15<M<0.75 \mathrm{MeV} / c^{2}$ from PHENIX published result [6], STAR preliminary result [8], and PHENIX preliminary result with the new HBD detector [14].

Figure 5 shows the PHENIX cocktail and the STAR cocktail filtered with the PHENIX azimuthal angle acceptance in $p+p 200 \mathrm{GeV}$ collisions. The two simulations match quite well, with some minor differences in the $\eta$ and $J / \psi$ regions that are due to the choice of slightly different form factors for $\eta$ decays and the different mass resolution between the two experiments. Therefore, the difference in the low mass region between PHENIX and STAR results cannot be attributed to a difference in cocktail simulations either.

PHENIX had a subsystem upgrade - the Hadron Blind Detector (HBD) in the last few years, aiming to further improve the signal-to-background $(S / B)$ ratio by rejecting conversions and $\pi^{0}$ Dalitz decays. The result with the HBD detector in the $\mathrm{p}+\mathrm{p}$ collisions reproduced the previously published result with a better $S / B$ ratio as expected. PHENIX has carried out the analysis with the HBD into the $\mathrm{Au}+\mathrm{Au}$ collisions and the results from mid-central to peripheral collisions were reported [14]. Figure 6 summarizes enhancement factors in the dielectron mass spectra in the mass region of $0.15-0.75 \mathrm{GeV} / \mathrm{c}^{2}$ with respect to the hadronic cocktail from PHENIX published result, STAR preliminary result and PHENIX preliminary result with the HBD. One can see the difference between previously published PHENIX results and the STAR results is mainly in the most central collisions. The new PHENIX HBD result is quite consistent with the earlier published PHENIX results as well as the STAR results from mid-central to peripheral collisions. One would be looking forward to the new PHENIX result with the HBD in the most central collisions soon.

\section{Dielectron Production at RHIC Beam Energy Scan}

RHIC launched the Beam Energy Scan (BES) program in the year 2010 aiming to map out the detailed QCD phase structure in a wide $T-\mu_{B}$ region. This offers a great opportunity to systematically investigate how the medium properties evolve with the change of the collision energy from the SPS up to the top RHIC energy. Preliminary results on the dielectron yields have been reported by the STAR experiment from Au+Au collisions for $\sqrt{s_{N N}}=19.6,39,62.4 \mathrm{GeV}$. In the low mass region, the observed dielectron yields compared to the hadronic cocktail (solid lines) yield a 


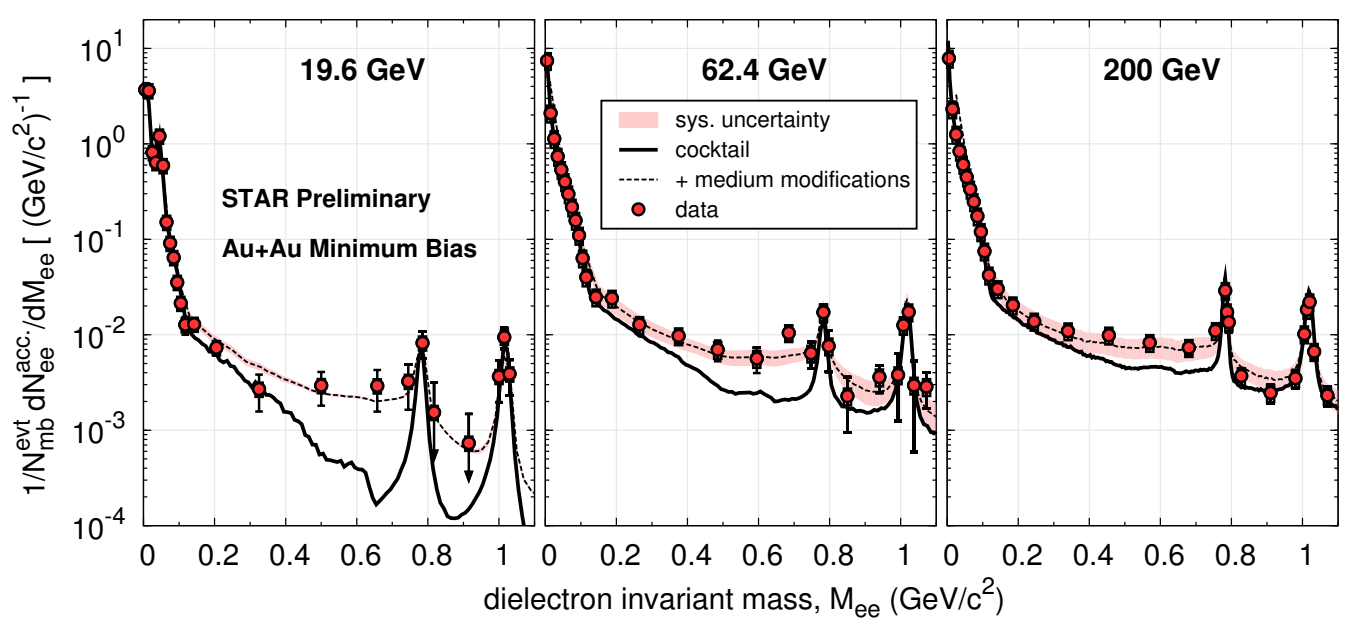

Figure 7: (Color online) Dielectron mass spectra in the STAR detector acceptance from $\mathrm{Au}+\mathrm{Au}$ minimum bias collisions at $\sqrt{s_{\mathrm{NN}}}=19.6,62.6$ and $200 \mathrm{GeV}$. The data are compared to the cocktail and the in-medium contribution from the many-body theory model [].

consistent enhancement in all energies, as shown in Fig. 7. Also shown in the figure are total sums (dashed lines) including model calculations for the medium contribution from Rapp [9]. The model calculations include both hadronic ( $\rho$-meson interacting with the hadronic medium) and partonic (QGP thermal radiation) contributions, which reproduce the excess observed in the data compared to the hadronic cocktail across a broad range of collision energies.

\section{Search for the QGP Thermal Radiation}

Thermal dileptons from the partonic medium radiation have been suggested as a clean signature of the QGP formation [1]. Theoretical calculations suggest that at RHIC energy, QGP thermal dilepton production will become dominant at $M_{l l}>1 \mathrm{GeV} / c^{2}$, and the higher the mass is, the earlier stage the thermal dileptons come from [2]. In Fig. 8 left panel, a direct comparison is shown between $0-80 \%$ minimum bias and $0-10 \%$ most central collisions. They are arbitrarily normalized in the $\pi^{0}$ mass region. In the mass region between $1-3 \mathrm{GeV} / c^{2}$, the mass spectrum shape in central collisions seems to become steeper than that in minimum bias collisions ( $1.5 \sigma$ difference in the slope by exponential fits). Therefore, correlated charm spectra from PYTHIA scaled with $N_{\text {bin }}$ cannot describe both the central and minimum bias data simultaneously. STAR further analyzed the slope parameter of the full acceptance corrected transverse mass spectra [8]. Figure 8 shows the slope parameters for inclusive dielectrons vs. their masses from $\mathrm{Au}+\mathrm{Au}$ and $p+p$ collisions. One can see that the $p+p$ slope parameters are consistent with the correlated charm contributions from PYTHIA calculations, while in $\mathrm{Au}+\mathrm{Au}$ collisions, the slope parameter at $2-3 \mathrm{GeV} / \mathrm{c}^{2}$ seems to be higher than in $p+p$ collisions. These observations may indicate that there could be modifications to the charm correlations or possibly another contributing source (e.g. QGP radiation) in Au + Au collisions at RHIC. 

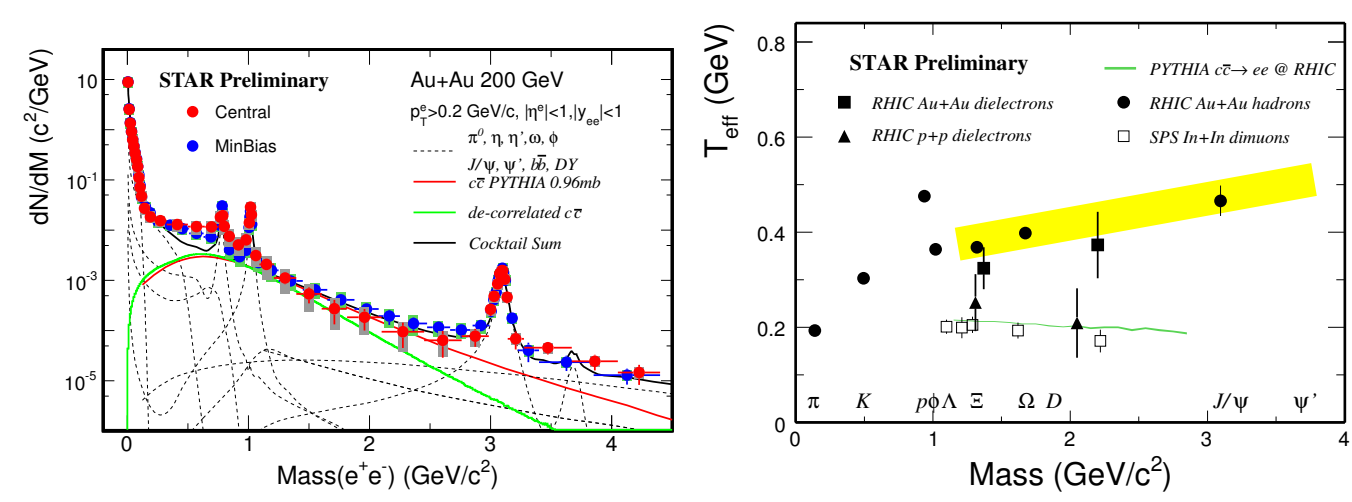

Figure 8: (Color online) Left: Direct comparison of dielectron mass spectra in minimum bias and 0-10\% central Au + Au collisions at $200 \mathrm{GeV}$ from STAR. Right: Slope parameter of transverse mass spectra from STAR inclusive dielectron measurement in $\mathrm{Au}+\mathrm{Au}$ and $p+p$ collisions compared to other RHIC hadron measurements and SPS dimuon measurements.

\section{Summary and Outlook}

Dilepton production serves as a sensitive probe to study the chiral and thermal properties of QCD medium. Recent measurements from RHIC experiments show an enhancement in the dielectron spectra in the mass region between $0.3-0.7 \mathrm{GeV} / c^{2}$ with respect to the hadronic cocktail, which is similar to what was observed in SPS dilepton data. However, the enhancement factors reported for the PHENIX and the STAR detector acceptance are significantly different for central and low $p_{T}$ dielectrons. Several experimental effects have been investigated and it is not likely that detector acceptance or cocktail simulation can account for this difference. STAR reported dielelectron spectra from the beam energy scan data. The enhancement factor in the low mass region persists from $\sqrt{s_{N N}}=19.6$ to $200 \mathrm{GeV}$. An effective many-body theory model calculation describes the observed excess in all energies with the in-medium modified $\rho$ spectral functions. The spectrum in the intermediate mass region from a couple of observations seems to indicate significant modifications to the charm correlations or possibly another contributing source in $\mathrm{Au}+$ $\mathrm{Au}$ collisions at $200 \mathrm{GeV}$. However, the uncertainty in the data is still large.

In the near future, PHENIX's new measurement with the HBD detector in central $\mathrm{Au}+\mathrm{Au}$ collisions is anticipated by the community. The Heavy Flavor Tracker and the Muon Telescope Detector updates at STAR will enable the direct measurement of charm correlations in heavy ion collisions. Systematic measurements from different collision energies offered by the flexible RHIC machine will allow detailed investigation of the onset of QGP thermal radiation.

\section{References}

[1] E. Shuryak, Phys. Rep. 61, 71 (1980).

[2] R. Rapp, Phys. Rev. C 63, 054907 (2001).

[3] E. Shuryak, arXiv: 1203.1012.

[4] R. Rapp and J. Wambach, Adv. Nucl. Phys. 25, 1 (2000).

[5] R. Arnaldi et al. [NA60 Collaboration], Phys. Rev. Lett. 96, 162302 (2006). 
[6] A. Adare et al. [PHENIX Collaboration], Phys. Rev. C 81, 034911 (2010).

[7] L. Adamczyk et al. [STAR Collaboration], Phys. Rev. C 86, 024906 (2012).

[8] J. Zhao (for STAR Collaboration), J. Phys. G 38, 124134 (2011).

[9] H. van Hees and R. Rapp, Phys. Rev. Lett. 97, 102301 (2006); H. van Hees and R. Rapp, Nucl. Phys. A 806, 339 (2008); R. Rapp, private communications.

[10] H.J. Xu et al., Phys. Rev. C 85, 024906 (2012).

[11] G. Gujanovic et al., arXiv: 1211.0022.

[12] W. Cassing and E.L. Bratkovskaya, Nucl. Phys. A 831, 215 (2009); E.L. Bratkovskaya, W. Cassing, V.P. Konchakovski, and O. Linnyk, Nucl. Phys. A 856, 162 (2011); O. Linnyk et al., Phys. Rev. C 85, 024910 (2012).

[13] J. Zhao (for STAR Collaboration), proceedings of Hard Probes 2012 conference, Cagliari, Italy, 2012.

[14] I. Tserruya (for PHENIX Collaboration), Nucl. Phys. A 904, 225c (2013).

[15] B. Huang (for STAR Collaboration), Nucl. Phys. A 904, 565c (2013). 